\title{
More Counterexamples to the Alon-Saks-Seymour and Rank-Coloring Conjectures
}

\author{
Sebastian M. Cioabă \\ Department of Mathematical Sciences \\ University of Delaware \\ Newark, DE 19716, USA \\ cioaba@math.udel.edu
}

\author{
Michael Tait ${ }^{\dagger}$ \\ Department of Mathematical Sciences \\ University of Delaware \\ Newark, DE 19716, USA \\ tait@math.udel.edu
}

Submitted: Nov 18, 2010; Accepted: Jan 25, 2011; Published: Feb 4, 2011

Mathematics Subject Classifications: 05C15, 05C50, 15A18

\begin{abstract}
The chromatic number $\chi(G)$ of a graph $G$ is the minimum number of colors in a proper coloring of the vertices of $G$. The biclique partition number $\operatorname{bp}(G)$ is the minimum number of complete bipartite subgraphs whose edges partition the edge-set of $G$.

The Rank-Coloring Conjecture (formulated by van Nuffelen in 1976) states that $\chi(G) \leq \operatorname{rank}(A(G))$, where $\operatorname{rank}(A(G))$ is the rank of the adjacency matrix of $G$. This was disproved in 1989 by Alon and Seymour. In 1991, Alon, Saks, and Seymour conjectured that $\chi(G) \leq \operatorname{bp}(G)+1$ for any graph $G$. This was recently disproved by Huang and Sudakov. These conjectures are also related to interesting problems in computational complexity.

In this paper, we construct new infinite families of counterexamples to both the Alon-Saks-Seymour Conjecture and the Rank-Coloring Conjecture. Our construction is a generalization of similar work by Razborov, and Huang and Sudakov.
\end{abstract}

\section{Introduction}

Our graph theoretic notation is standard (see West [20]). In this paper, all the graphs are simple and undirected. The biclique partition number $\operatorname{bp}(G)$ of a graph $G$ is the minimum number of complete bipartite subgraphs (also called bicliques) whose edges partition the edge set of $G$. The chromatic number $\chi(G)$ is the minimum number of colors needed in

*The author's research was supported by a start-up grant from the Department of Mathematical Sciences of University of Delaware.

†This paper is part of the author's M.Sc. Thesis. 
a proper coloring of the vertices of $G$. The adjacency matrix $A(G)$ of $G$ has its rows and columns indexed after the vertices of $G$ and its $(u, v)$-th entry equals 1 if the vertices $u$ and $v$ are adjacent in $G$ and 0 otherwise. The rank of $A(G)$ will be denoted by $\operatorname{rank}(A(G))$.

Motivated by network design problems, Graham and Pollak [7] proved that the edgeset of a complete graph on $n$ vertices cannot be partitioned into fewer than $n-1$ bicliques. This result can be restated as $\chi\left(K_{n}\right)=\mathrm{bp}\left(K_{n}\right)+1$. Over the years, several proofs of this fact have been discovered (see $[13,17,18,19]$ ). A natural generalization of the GrahamPollak Theorem is to ask if any graph $G$ can be properly colored with $\operatorname{bp}(G)+1$ colors. This question was first posed by Alon, Saks, and Seymour (cf. Kahn [9]) .

Conjecture 1.1 (Alon-Saks-Seymour). For any simple graph $G, \chi(G) \leq \mathrm{bp}(G)+1$.

This conjecture was confirmed by Rho [15] for graphs $G$ with $n$ vertices and $\operatorname{bp}(G) \in$ $\{1,2,3,4, n-3, n-2, n-1\}$ and by Gao, McKay, Naserasr and Stevens [6] for graphs with $\operatorname{bp}(G) \leq 9$. The Alon-Saks-Seymour Conjecture remained open for twenty years until recently when Huang and Sudakov [8] constructed the first counterexamples.

In 1976, van Nuffelen [12] (see also Fajtlowicz [5]) stated what became known as the Rank-Coloring Conjecture.

Conjecture 1.2 (Rank-Coloring). For any simple graph $G, \chi(G) \leq \operatorname{rank}(A(G))$.

The Rank-Coloring Conjecture was disproved in 1989 by Alon and Seymour [1]. Razborov [14] found counterexamples with a superlinear gap between $\operatorname{rank}(A(G))$ and $\chi(G)$. Other counterexamples were constructed from the Kasami graphs by Roy and Royle [16]. To our knowledge, Nisan and Wigderson's construction from [11] yields the largest gap between the chromatic number and the rank at present time. The Alon-SaksSeymour Conjecture and the Rank-Coloring Conjecture are closely related to computational complexity problems (see $[8,10,11]$ ).

In this paper, we construct infinitely many graphs that are counterexamples to both the Alon-Saks-Seymour Conjecture and the Rank-Coloring Conjecture. More precisely, we construct infinite families of graphs $G(n, k, r)$ with $n^{2 k+2 r+1}$ vertices for all integers $n \geq 2, k \geq 1, r \geq 1$ such that

$$
\chi(G(n, k, r)) \geq \frac{n^{2 k+2 r}}{2 r+1}
$$

and for $k \geq 2$

$$
2 k(2 r+1)(n-1)^{2 k+2 r-1} \leq \mathrm{bp}(G(n, k, r))<2^{2 k+2 r-1} n^{2 k+2 r-1}
$$

and

$$
2 k(2 r+1)(n-1)^{2 k+2 r-1} \leq \operatorname{rank}(A(G(n, k, r)))<2 k(2 r+1) n^{2 k+2 r-1}
$$

These inequalities imply that for fixed $k \geq 2$ and $r \geq 1$ and $n$ large enough, the graphs $G(n, k, r)$ are counterexamples to both the Alon-Saks-Seymour Conjecture and the Rank-Coloring Conjecture. Our construction extends the constructions of Huang and 
Sudakov [8] and Razborov [14]. Taking $k=2$ and $r=1$, we get Huang and Sudakov's graph sequence from [8]. When $k=1$ and $r=1$, we obtain Razborov's construction from [14].

In Section 2, we describe the construction of the graphs $G(n, k, r)$ and we prove inequality (1) and the upper bound on $\operatorname{bp}(G(n, k, r))$ from (2). In Section 3, we obtain the bounds (3) on the rank of the adjacency matrix of $G(n, k, r)$ and deduce the lower bound on $\operatorname{bp}(G(n, k, r))$ from $(2)$.

\section{The graphs $G(n, k, r)$}

Let $Q_{n}$ be the $n$-dimensional cube with vertex set $\{0,1\}^{n}$ and two vertices $x, y$ in $Q_{n}$ adjacent if and only if they differ in exactly one coordinate. A $k$-dimensional subcube of $Q_{n}$ is a subset of $Q_{n}$ which can be written as

$$
\left\{x=\left(x_{1}, \ldots, x_{n}\right) \in Q_{n} \mid x_{i}=b_{i}, \forall i \in B\right\}
$$

where $B$ is a set of $n-k$ fixed coordinates and each $b_{i} \in\{0,1\}$. We represent the all ones and all zeros vectors as $1^{n}$ and $0^{n}$ respectively, and we define $Q_{n}^{-}=Q_{n} \backslash\left\{1^{n}, 0^{n}\right\}$. For any integer $n \geq 1$, we denote $\{1, \ldots, n\}$ by $[n]$.

For given integers $n \geq 2, k \geq 1$, and $r \geq 1$, we define the graph $G(n, k, r)$ as follows. Its vertex set is

$$
V(G(n, k, r))=[n]^{2 k+2 r+1}=\left\{\left(x_{1}, \ldots, x_{2 k+2 r+1}\right) \mid x_{i} \in[n], \forall i, 1 \leq i \leq 2 k+2 r+1\right\} .
$$

For any two vertices $x=\left(x_{1}, \ldots, x_{2 k+2 r+1}\right), y=\left(y_{1}, \ldots, y_{2 k+2 r+1}\right)$ let

$$
\rho(x, y)=\left(\rho_{1}(x, y), \ldots, \rho_{2 k+2 r+1}(x, y)\right) \in\{0,1\}^{2 k+2 r+1}
$$

where $\rho_{i}(x, y)=1$ if $x_{i} \neq y_{i}$ and $\rho_{i}(x, y)=0$ if $x_{i}=y_{i}$.

We define adjacency in $G(n, k, r)$ as follows: the vertices $x$ and $y$ are adjacent in $G(n, k, r)$ if and only if $\rho(x, y) \in S$ where

$$
S=Q_{2 k+2 r+1} \backslash\left[\left(1^{2 k} \times Q_{2 r+1}^{-}\right) \cup\left\{0^{2 k} \times 0^{2 r+1}\right\} \cup\left\{0^{2 k} \times 1^{2 r+1}\right\}\right]
$$

We will prove now the lower bound (1) for the chromatic number of $G(n, k, r)$.

Proposition 2.1. For $n \geq 2$ and $k, r \geq 1, \chi(G(n, k, r)) \geq \frac{n^{2 k+2 r}}{2 r+1}$.

Proof. In this proof we will refer to $G(n, k, r)$ as $G$.

For $x=\left(x_{1}, \ldots, x_{2 k}, x_{2 k+1}, \ldots, x_{2 k+2 r+1}\right) \in V(G)$, let $f(x)=\left(x_{1}, \ldots, x_{2 k}\right)$ be the projection to the first $2 k$ coordinates of $x$ and $t(x)=\left(x_{2 k+1}, \ldots, x_{2 k+2 r+1}\right)$ be the projection to the last $2 r+1$ coordinates of $x$.

Let $I$ be an independent set in $G$. Any two vertices $x$ and $y$ of $G$ which agree on one of the first $2 k$ coordinates and satisfy $f(x) \neq f(y)$ are adjacent in $G$. This implies that any two distinct vectors in $f(I)$ differ in all of the first $2 k$ of their coordinates and thus, $|f(I)| \leq n$. 
If for every $u \in f(I),\left|f^{-1}(u) \cap I\right| \leq 2 r+1$, then $|I| \leq(2 r+1) n$. Otherwise, there is a $\beta \in[n]^{2 k}$ and distinct $x_{1}, x_{2}, \ldots, x_{2 r+2} \in I$ such that $f\left(x_{i}\right)=\beta$ for $1 \leq i \leq 2 r+2$. Then $\rho\left(t\left(x_{i}\right), t\left(x_{j}\right)\right)=1^{2 r+1}$ for any $1 \leq i \neq j \leq 2 r+2$. From the definition (6) of $S$, we know that any two vertices that differ in all $2 k+2 r+1$ coordinates are adjacent in $G$. If there exists a $z \in I$ such that $f(z)$ and $\beta$ differ on every coordinate, then $t(z)$ and $t\left(x_{i}\right)$ are equal in at least one coordinate for each $i$. Thus at least two of $x_{1}, x_{2}, \ldots, x_{2 r+2}$ must agree in at least one coordinate of $t(z)$, contradicting that $t\left(x_{i}\right)$ must differ in every coordinate for distinct $i$. Thus, there must be only one element in $f(I)$. Again, the vertices in $I$ must differ in all of the last $2 r+1$ coordinates, and thus $|I|=|f(I)| \leq n$.

Thus, we proved that the independence number of $G$ satisfies the inequality $\alpha(G) \leq$ $(2 r+1) n$. This fact and $\chi(G) \geq \frac{|V(G)|}{\alpha(G)}$ complete our proof.

To prove the upper bound (2) on the biclique partition number of $G(n, k, r)$, we need some auxiliary lemmas.

Lemma 2.2. The set $Q_{2 k+1}^{-}$can be partitioned into a disjoint union of 1-dimensional subcubes for $k \geq 1$.

Proof. We prove the lemma by induction on $k$.

In the base case when $k=1$, we can write

$$
Q_{3}^{-}=\{(0,0,1),(0,1,1)\} \cup\{(0,1,0),(1,1,0)\} \cup\{(1,0,0),(1,0,1)\} .
$$

This proves the base case.

Assume now that $Q_{2 k+1}^{-}$can be partitioned into 1-dimensional subcubes. Then

$$
\begin{aligned}
Q_{2 k+3} & =\left(Q_{2 k+1} \times 1 \times 0\right) \cup\left(Q_{2 k+1} \times 1 \times 1\right) \cup\left(Q_{2 k+1} \times 0 \times 1\right) \cup\left(Q_{2 k+1} \times 0 \times 0\right) \\
& =\left(Q_{2 k+1} \times 1 \times 0\right) \\
& \cup\left(Q_{2 k+1}^{-} \times 1 \times 1 \cup\left\{1^{2 k+1} \times 1 \times 1\right\} \cup\left\{0^{2 k+1} \times 1 \times 1\right\}\right) \\
& \cup\left(Q_{2 k+1}^{-} \times 0 \times 1 \cup\left\{1^{2 k+1} \times 0 \times 1\right\} \cup\left\{0^{2 k+1} \times 0 \times 1\right\}\right) \\
& \cup\left(Q_{2 k+1}^{-} \times 0 \times 0 \cup\left\{1^{2 k+1} \times 0 \times 0\right\} \cup\left\{0^{2 k+1} \times 0 \times 0\right\}\right)
\end{aligned}
$$

This implies

$$
\begin{aligned}
Q_{2 k+3}^{-} & =\left(Q_{2 k+1} \times 1 \times 0\right) \\
& \cup\left(Q_{2 k+1}^{-} \times 1 \times 1 \cup\left\{0^{2 k+1} \times 1 \times 1\right\}\right) \\
& \cup\left(Q_{2 k+1}^{-} \times 0 \times 1 \cup\left\{1^{2 k+1} \times 0 \times 1\right\} \cup\left\{0^{2 k+1} \times 0 \times 1\right\}\right) \\
& \cup\left(Q_{2 k+1}^{-} \times 0 \times 0 \cup\left\{1^{2 k+1} \times 0 \times 0\right\}\right)
\end{aligned}
$$

which equals

$$
\begin{gathered}
\left(Q_{2 k+1} \times 1 \times 0\right) \cup\left(Q_{2 k+1}^{-} \times 1 \times 1\right) \cup\left(Q_{2 k+1}^{-} \times 0 \times 1\right) \cup\left(Q_{2 k+1}^{-} \times 0 \times 0\right) \cup \\
\left\{1^{2 k+1} \times 0 \times 1,1^{2 k+1} \times 0 \times 0\right\} \cup\left\{0^{2 k+1} \times 1 \times 1,0^{2 k+1} \times 0 \times 1\right\} .
\end{gathered}
$$

By induction hypothesis, it follows that $Q_{2 k+3}^{-}$can be partitioned into 1-dimensional subcubes. 
We use the previous lemma to prove that the set $S$ defined in (6) can be partitioned into 2-dimensional subcubes.

Lemma 2.3. For $k \geq 2$ and $r \geq 1$, the set

$$
S=Q_{2 k+2 r+1} \backslash\left[\left(1^{2 k} \times Q_{2 r+1}^{-}\right) \cup\left\{0^{2 k} \times 0^{2 r+1}\right\} \cup\left\{0^{2 k} \times 1^{2 r+1}\right\}\right]
$$

can be partitioned into 2-dimensional subcubes.

Proof. We claim that the following three sets form a partition of $S$ :

$$
\begin{gathered}
S^{\prime}=\left(0^{2 k-1} \times 0 \times Q_{2 r+1}^{-}\right) \cup\left(0^{2 k-1} \times 1 \times Q_{2 r+1}^{-}\right) \cup\left(Q_{2 k-1}^{-} \times 1 \times Q_{2 r+1}^{-}\right) \\
S^{\prime \prime}=\left(Q_{2 k-1} \times 1 \times 0^{2 r+1}\right) \cup\left(Q_{2 k-1} \times 1 \times 1^{2 r+1}\right)
\end{gathered}
$$

and

$$
S^{\prime \prime \prime}=\left(Q_{2 k-1} \backslash\left\{0^{2 k-1}\right\}\right) \times 0 \times Q_{2 r+1} .
$$

To show this is a partition, we first prove $S \subseteq S^{\prime} \cup S^{\prime \prime} \cup S^{\prime \prime \prime}$. To see this, consider the $2 k$-th coordinate of any vector $s=\left(s_{1}, \ldots, s_{2 k+2 r+1}\right)$ in $S$. As before, let $f(s)=\left(s_{1}, \ldots, s_{2 k}\right)$ and $t(s)=\left(s_{2 k+1}, \ldots, s_{2 k+2 r+1}\right)$. If $s_{2 k}=0$, and $f(s) \neq 0^{2 k}$, then $s \in S^{\prime \prime \prime}$. If $f(s)=0^{2 k}$ then $s \in S^{\prime}$. Now take $s \in S$ such that $s_{2 k}=1$. If $t(s)=1^{2 r+1}$ or $t(s)=0^{2 r+1}$, then $s \in S^{\prime \prime}$. Otherwise, $s \in S^{\prime}$. Thus $S \subseteq S^{\prime} \cup S^{\prime \prime} \cup S^{\prime \prime \prime}$. Since $S^{\prime}, S^{\prime \prime}, S^{\prime \prime \prime}$ are disjoint subsets of $S$, they must partition $S$.

The set $Q_{2 r+1}$ can be partitioned into 2-dimensional subcubes. It follows that for any $\beta \in Q_{2 k}$, the set $\beta \times Q_{2 r+1}$ can also be partitioned into 2-dimensional subcubes. For any $x_{1}$ adjacent to $x_{2}$ in $Q_{2 k}, y_{1}$ adjacent to $y_{2}$ in $Q_{2 r+1}$, the set $\left\{\left(x_{1}, y_{1}\right),\left(x_{1}, y_{2}\right),\left(x_{2}, y_{1}\right),\left(x_{2}, y_{2}\right)\right\}$ is a 2-dimensional subcube. By Lemma 2.2, $Q_{2 r+1}^{-}$can be decomposed into 1-dimensional subcubes. This implies that for any $x_{1}$ adjacent to $x_{2}$ in $Q_{2 k},\left(x_{1} \times Q_{2 r+1}^{-}\right) \cup\left(x_{2} \times Q_{2 r+1}^{-}\right)$ can be decomposed into 2-dimensional subcubes.

These remarks imply that $S^{\prime}, S^{\prime \prime}, S^{\prime \prime \prime}$ and thus $S$ can be partitioned into 2-dimensional subcubes.

Using the previous lemma, we are ready to prove the upper bound (2) for the biclique partition number of the graph $G(n, k, r)$.

Proposition 2.4. For $n \geq 2, k \geq 2, r \geq 1, \operatorname{bp}(G(n, k, r))<2^{2 k+2 r-1} n^{2 k+2 r-1}$.

Proof. In this proof we will refer to $G(n, k, r)$ as $G$.

By Lemma 2.3, $S=\cup_{i=1}^{t} S_{i}$, where $t=\frac{2^{2 k+2 r+1}-2^{2 r+1}}{4}=2^{2 k+2 r-1}-2^{2 r-1}$ and each $S_{i}$ is a 2-dimensional subcube. For $1 \leq i \leq t$, let $G_{i}$ be the subgraph of $G$ such that $x, y \in V\left(G_{i}\right)=V(G)=[n]^{2 k+2 r+1}$ are adjacent if and only if $\rho(x, y) \in S_{i}$. Then the edge sets of the subgraphs $G_{1}, G_{2}, \ldots, G_{t}$ partition the edge set of the graph $G$. For each $S_{i}$ there is a set $T_{i}=\left\{t_{1}, \ldots, t_{2 k+2 r-1}\right\} \subset\{1, \ldots, 2 k+2 r+1\}$ of fixed coordinates $a_{1}, \ldots, a_{2 k+2 r-1} \in\{0,1\}$ so that $S_{i}=\left\{\left(x_{1}, \ldots, x_{2 k+2 r+1}\right) \mid x_{t_{j}}=a_{j}, \forall 1 \leq j \leq 2 k+2 r-1\right\}$.

Define $G_{i}^{\prime}$ with vertex set $[n]^{2 k+2 r-1}$ such that $x^{\prime}$ and $y^{\prime}$ adjacent in $G_{i}^{\prime}$ if and only if $\rho\left(x^{\prime}, y^{\prime}\right)=\left(a_{1}, \ldots, a_{2 k+2 r-1}\right)$. Then $G_{i}$ is an $n^{2}$-blowup of $G_{i}^{\prime}$ which means that $G_{i}$ can be 
obtained from $G_{i}^{\prime}$ by replacing each vertex $v$ of $G_{i}^{\prime}$ by an independent set $I_{v}$ of $n^{2}$ vertices and by adding all edges between $I_{u}$ and $I_{v}$ in $G_{i}$ whenever $u$ and $v$ are adjacent in $G_{i}^{\prime}$. Note that a partition of $G_{i}^{\prime}$ into complete bipartite subgraphs becomes a partition into complete bipartite subgraphs in any blowup of $G_{i}^{\prime}$. Thus $\operatorname{bp}\left(G_{i}\right) \leq \operatorname{bp}\left(G_{i}^{\prime}\right) \leq\left|V\left(G_{i}^{\prime}\right)\right|-1 \leq$ $n^{2 k+2 r-1}-1$. Since the edge set of $G$ is the disjoint union of the edge sets of $G_{1}, \ldots, G_{t}$, we have that

$$
\operatorname{bp}(G) \leq \sum_{i=1}^{t} \operatorname{bp}\left(G_{i}\right) \leq\left(2^{2 k+2 r-1}-2^{2 r-1}\right)\left(n^{2 k+2 r-1}-1\right)<2^{2 k+2 r-1} n^{2 k+2 r-1} .
$$

\section{The rank of $A(G(n, k, r))$}

In this section, we obtain asymptotically tight bounds for the rank of the adjacency matrix of $G(n, k, r)$. We will use the following graph operation called NEPS (Non-complete Extended P-Sum) introduced by Cvetković in his thesis [3] (see also [4] page 66).

Definition 3.1. For given $\mathcal{B} \subset\{0,1\}^{t} \backslash\left\{0^{t}\right\}$ and graphs $G_{1}, \ldots, G_{t}$, the NEPS with basis $\mathcal{B}$ of the graphs $G_{1}, \ldots, G_{t}$ is the graph whose vertex set is the cartesian product of the sets of vertices of the graphs $G_{1}, \ldots, G_{t}$ and in which two vertices $\left(x_{1}, \ldots, x_{t}\right)$ and $\left(y_{1}, \ldots, y_{t}\right)$ are adjacent if and only if there is a t-tuple $\left(b_{1}, \ldots, b_{t}\right)$ in $\mathcal{B}$ such that $x_{i}=y_{i}$ holds exactly when $b_{i}=0$ and $x_{i}$ is adjacent to $y_{i}$ in $G_{i}$ exactly when $b_{i}=1$.

Note that when all the graphs $G_{1}, \ldots, G_{t}$ are isomorphic to the complete graph $K_{n}$, then the NEPS with basis $\mathcal{B}$ of $G_{1}, \ldots, G_{t}$ will be the graph whose vertex set is $[n]^{t}$ with $\left(x_{1}, \ldots, x_{t}\right) \sim\left(y_{1}, \ldots, y_{t}\right)$ if and only if $\rho\left(\left(x_{1}, \ldots, x_{t}\right),\left(y_{1}, \ldots, y_{t}\right)\right)=\left(b_{1}, \ldots, b_{t}\right)$ for some $\left(b_{1}, \ldots, b_{t}\right) \in \mathcal{B}$.

Hence, the graph $G(n, k, r)$ is the NEPS of $2 k+2 r+1$ copies of $K_{n}$ with basis

$$
S=Q_{2 k+2 r+1} \backslash\left[\left(1^{2 k} \times Q_{2 r+1}^{-}\right) \cup\left\{0^{2 k} \times 0^{2 r+1}\right\} \cup\left\{0^{2 k} \times 1^{2 r+1}\right\}\right] .
$$

Another important observation (see Theorem 2.21 on page 68 in [4]) is that the adjacency matrix of the NEPS with basis $\mathcal{B}$ of $G_{1}, \ldots, G_{t}$ equals

$$
\sum_{\left(b_{1}, \ldots, b_{t}\right) \in \mathcal{B}} A\left(G_{1}\right)^{b_{1}} \otimes \cdots \otimes A\left(G_{t}\right)^{b_{t}}
$$

where $X \otimes Y$ denotes the Kronecker product of two matrices $X$ and $Y$.

These facts will enable us to compute the eigenvalues of $G(n, k, r)$ and to obtain the bounds from (3) on the rank of the adjacency matrix of $G(n, k, r)$.

Proposition 3.2. For $n \geq 2, k \geq 1, r \geq 1$,

$$
2 k(2 r+1)(n-1)^{2 k+2 r-1} \leq \operatorname{rank}(A(G(n, k, r)))<2 k(2 r+1) n^{2 k+2 r-1} .
$$


Proof. By Theorem 2.23 on page 69 in [4] or by the previous observations, the spectrum of the adjacency matrix of $G(n, k, r)$ has the following form:

$$
\Lambda(G)=\left\{f\left(\lambda_{1}, \ldots, \lambda_{2 k+2 r+1}\right) \mid \lambda_{1}, \ldots, \lambda_{2 k+2 r+1} \text { eigenvalues of } K_{n}\right\}
$$

where

$$
f\left(x_{1}, \ldots, x_{2 k+2 r+1}\right)=\sum_{\left(s_{1}, \ldots, s_{2 k+2 r+1}\right) \in S} \prod_{i=1}^{2 k+2 r+1} x_{i}^{s_{i}}
$$

Using the definition of $S$, we can simplify $f\left(x_{1}, \ldots, x_{2 k+2 r+1}\right)$ as follows

$f\left(x_{1}, \ldots, x_{2 k+2 r+1}\right)=\prod_{i=1}^{2 k+2 r+1}\left(1+x_{i}\right)-1-\prod_{i=1}^{2 k} x_{i}\left[\prod_{i=2 k+1}^{2 k+2 r+1}\left(1+x_{i}\right)-1-\prod_{i=2 k+1}^{2 k+2 r+1} x_{i}\right]-\prod_{i=2 k+1}^{2 k+2 r+1} x_{i}$.

Whenever the last $2 r+1$ positions are $-1, f$ evaluates as

$$
f\left(x_{1}, \ldots, x_{2 k},-1, \ldots,-1\right)=-1-\prod_{i=1}^{2 k} x_{i}\left[-1-(-1)^{2 r+1}\right]-(-1)^{2 r+1}=0 .
$$

Whenever the first $2 k$ positions are $-1, f$ evaluates as

$$
f\left(-1, \ldots,-1, x_{2 k+1}, \ldots, x_{2 k+2 r}\right)=-1-(-1)^{2 k}\left[-1-\prod_{i=2 k+1}^{2 k+2 r+1} x_{i}\right]-\prod_{i=2 k+1}^{2 k+2 r+1} x_{i}=0 .
$$

Thus, we obtain 0 as an eigenvalue for $G(n, k, r)$ when all of the last $2 r+1$ positions are -1 or when the first $2 k$ positions are -1 . The eigenvalues of $K_{n}$ are $n-1$ with multiplicity 1 and -1 with multiplicity $n-1$. We will make use of the following simple inequality: $n^{t}-(n-1)^{t}<t n^{t-1}$ for any integers $n, t \geq 1$.

These facts imply that $G(n, k, r)$ has eigenvalue 0 with multiplicity at least

$$
\begin{aligned}
n^{2 k}(n-1)^{2 r+1}+ & (n-1)^{2 k} n^{2 r+1}-(n-1)^{2 k+2 r+1} \\
& =n^{2 k+2 r+1}-\left(n^{2 k}-(n-1)^{2 k}\right)\left(n^{2 r+1}-(n-1)^{2 r+1}\right) \\
& >n^{2 k+2 r+1}-2 k n^{2 k-1} \cdot(2 r+1) n^{2 r+1-1} \\
& =n^{2 k+2 r+1}-2 k(2 r+1) n^{2 k+2 r-1} .
\end{aligned}
$$

which shows that

$$
\operatorname{rank}(A(G(n, k, r)))<2 k(2 r+1) n^{2 k+2 r-1} .
$$

To prove the other part, note that for fixed $u \in\{1, . ., 2 k\}$ and $v \in\{2 k+1, . ., 2 k+2 r+1\}$, evaluating $f$ when $x_{i}=-1$ for $i \neq u, v$ (by using (13)), we get

$$
f\left(-1, \ldots, x_{u}, \ldots, x_{v}, \ldots,-1\right)=-1+x_{u}\left(-1-x_{v}\right)-x_{v}=-\left(x_{u}+1\right)\left(x_{v}+1\right) .
$$

If $x_{u}=x_{v}=n-1$, we obtain $f\left(-1, \ldots, x_{u}, \ldots, x_{v}, \ldots,-1\right)=-n^{2}$. Since $K_{n}$ has eigenvalue -1 with multiplicity $n-1$, we deduce that $G(n, k, r)$ has the negative eigenvalue $-n^{2}$ with multiplicity at least $\left(\begin{array}{c}2 k \\ 1\end{array}\right)\left(\begin{array}{c}2 r+1 \\ 1\end{array}\right)(n-1)^{2 k+2 r-1}$. This $\operatorname{shows} \operatorname{rank}(A(G(n, k, r))) \geq$ $2 k(2 r+1)(n-1)^{2 k+2 r-1}$ and completes our proof. 
A result of Witsenhausen (cf. Graham and Pollak [7]) states that for any graph $H$

$$
\operatorname{bp}(H) \geq \max \left(n_{+}(A(H)), n_{-}(A(H))\right)
$$

where $n_{+}(A(H))$ and $n_{-}(A(H))$ denote the number of positive and the number of negative eigenvalues of the adjacency matrix of $H$, respectively.

From the last part of the proof of Proposition 3.2, we deduce that $n_{-}(A(G(n, k, r))) \geq$ $2 k(2 r+1)(n-1)^{2 k+2 r-1}$. This result and inequality (18) imply

$$
\operatorname{bp}(G(n, k, r)) \geq 2 k(2 r+1)(n-1)^{2 k+2 r-1} .
$$

As $\operatorname{bp}(A(G(n, k, r))) \leq 2^{2 k+2 r-1} n^{2 k+2 r-1}$, this shows that $\operatorname{bp}(A(G(n, k, r)))=\Theta\left(n^{2 k+2 r-1}\right)$ for fixed $k \geq 2$ and $r \geq 1$.

\section{Conclusion}

In this paper, we constructed new families of counterexamples to the Alon-Saks-Seymour Conjecture and to the Rank-Coloring Conjecture. We computed the eigenvalues of the adjacency matrices of these graphs and obtained tight bounds for the rank of their adjacency matrices. We used these results to determine the asymptotic behavior of their biclique partition number. It would be interesting to determine other properties of these graphs.

It remains an open problem to see how large the gap between the biclique partition number and the chromatic number of a graph can be in general. At present time, Huang and Sudakov's construction from [8] gives the biggest gap between biclique partition number and chromatic number. Their construction yields an infinite sequence of graphs $G_{n}$ such that $\chi\left(G_{n}\right) \geq c\left(\operatorname{bp}\left(G_{n}\right)\right)^{6 / 5}$ for some fixed constant $c>0$. Huang and Sudakov conjecture in [8] that there exists a graph $G$ with biclique partition number $k$ and chromatic number at least $2^{c \log ^{2} k}$, for some constant $c>0$.

\section{Acknowledgments}

We thank the referee for some useful comments.

\section{References}

[1] N. Alon and P. Seymour, A counterexample to the rank-coloring conjecture, J. Graph Theory 13 (1989), no. 4, 523-525.

[2] A. Brouwer and W. Haemers, Spectra of Graphs, manuscript 229pp available online at http://homepages.cwi.nl/ aeb/math/ipm.pdf .

[3] D. Cvetković, Graphs and their spectra, Univ. Beograd, Publ. Elektrotehn. Fak. Ser. Mat. Fiz. 356 (1971), 1-50. 
[4] D.M. Cvetković, M. Doob and H. Sachs, Spectra of Graphs: Theory and Application Pure and Applied Mathematics, 87. Academic Press, New York-London, 1980. 368 pp.

[5] S. Fajtlowicz, On conjectures of Graffiti, II, Congressus Numeratium 60 (1987) 187197.

[6] Z. Gao, B.D. McKay, R. Naserasr and B. Stevens, On Alon-Saks-Seymour Conjecture, to appear.

[7] R.L. Graham and H.O. Pollak, On the addressing problem for loop switching, Bell Syst. Tech. J. 50 (8) (1971), 2495-2519.

[8] H. Huang and B. Sudakov, A counterexample to the Alon-Saks-Seymour conjecture and related problems, to appear.

[9] J. Kahn, Recent results on some not-so-recent hypergraph matching and covering problems, Extremal problems for finite sets (1991), Bolyai Soc. Math. Stud. Vol. 3, János Bolyai Math. Soc., Budapest, 1994, 305-353.

[10] L. Lovász and M. Saks, Lattices, Möbius functions, and communication complexity, Journal of Computer and System Sciences 47 (1993), 322-349.

[11] N. Nisan and A. Wigderson, On rank vs. communication complexity, Combinatorica 15 (1995), 557-565.

[12] C. van Nuffelen, Research Problems: A Bound for the Chromatic Number of a Graph, Amer. Math. Monthly 83 (1976), no. 4, 265-266.

[13] G. Peck, A new proof of a theorem of Graham and Pollak, Discrete Math. 49 (1984), 327-328.

[14] A. Razborov, The gap between the chromatic number of a graph and the rank of its adjacency matrix is superlinear, Discrete Math. 108 (1992) 393-396.

[15] Y. Rho, A note on the Alon-Saks-Seymour coloring conjecture, Ars Combin. 63 (2002), 289-292.

[16] A. Roy and G. Royle, The chromatic number and rank of the complements of the Kasami graphs, Discrete Math. 307 (2007), no. 1, 132-136.

[17] H. Tverberg, On the decomposition of $K_{n}$ into complete bipartite graphs, J. Graph Theory 6 (1982), 493-494.

[18] S. Vishwanathan, A polynomial space proof of the Graham-Pollak theorem, J. Combin. Theory Ser. A 115 (2008), 674-676.

[19] S. Vishwanathan, A counting proof of the Graham-Pollak theorem, available online at http://arxiv.org/pdf/1007.1553v1.

[20] D.B. West, Introduction to Graph Theory, Prentice Hall, Upper Saddle River, NJ (1996). 